\title{
Experience with insufflation technique in the management of incisional hernia with loss of domain
}

\author{
I Bombil, ${ }^{1}$ (D) L Lushiku² iD \\ ${ }^{1}$ Department of Surgery, University of the Witwatersrand, South Africa \\ ${ }^{2}$ Department of Anaesthesia, University of the Witwatersrand, South Africa
}

Corresponding author, email: ifongobombil@gmail.com

Background: The loss of intra-abdominal domain makes it impossible to reconstruct the abdominal wall defect without prior correction of container/content mismatch. This study reports on the use of an insufflation technique to enable repair. Method: Over an 8-year period, 19 incisional hernia patients with loss of domain between $20 \%$ and $50 \%$ were treated with an insufflation technique to create sufficient intra-abdominal domain to effect repair. Ambient air was insufflated into the abdominal cavity via an indwelling catheter. After progressive pneumoperitoneum was completed over a period of 10 to 14 days, the hernias were repaired.

Results: There were 16 females and three males. The defect size ranged from $7-25 \mathrm{~cm}$. The mean air insufflated was 7.2 litres. After pneumoperitoneum, approximation of the linea alba was possible in all cases with a mean intraoperative predeflation and post-closure peak airway pressure within normal (21 and $20 \mathrm{cmH}_{2} \mathrm{O}$ respectively). Tissue repair and implant reinforcement were performed in 17 and two cases respectively. There were three instances of surgical site complications and no 30-day recurrence.

Conclusion: Repair of incisional hernia with significant loss of domain was made possible by the insufflation technique that enabled tension-free closure of the abdominal wall defect by direct suture, evidenced by the normal peak airway pressure after closure.

Keywords: insufflation technique, incisional hernia, complications of laparotomy

\section{Introduction}

Incisional hernias (IH) are one of the most common complications of laparotomy and a subset is associated with significant loss of domain (LOD). ${ }^{1,2}$ This is due to a lack of approximation of the linea alba resulting in retraction of the abdominal wall musculature and the herniation of intraabdominal viscera extra-abdominally, a situation exacerbated by visceral oedema. ${ }^{3}$ The end result is a reduction of the capacity of the container (the intra-abdominal space) for the volume of the content. The container represents the volume of the abdominal cavity, and the content is the volume of intra-abdominal viscera. The conventional approach consists of approximating the midline under tension, with high risk of compartment syndrome and failure of the repair if the container/content mismatch is not addressed. ${ }^{3}$ The strategy to re-expand the volume of the abdominal cavity combined with the concomitant reduction of the volume of the intra-abdominal viscera is the goal of the insufflation technique (IT) using progressive pneumoperitoneum (PPP). PPP is an old technique that was first described by Goni Moreno in the 1960s. Ambient air is insufflated daily via an abdominal catheter over a 10-14-day period. The abdominal catheter can be placed either blindly with the use of a Seldinger technique, under sonar guidance with open surgery, or laparoscopically. ${ }^{1,3-7}$ The PPP is not without complications. Besides the compartment syndrome, there is a potential risk of deep vein thrombosis (DVT) that can result in fatal pulmonary embolism (PE), making DVT prophylaxis mandatory. ${ }^{1,8}$ This study aims at exploring our local experience with the use of insufflation technique to achieve a tension-free closure of IHs with significant LOD and to reflect on the short-term outcomes of this approach.

\section{Methods}

This study is a retrospective case series of IH with significant LOD (between 20\% and 50\%) treated with IT from January 2013 to May 2020 at Chris Hani Baragwanath Academic Hospital (CHBAH) in Johannesburg. Patients unfit for surgery and the cases with more than two recurrences were excluded. All patients received DVT prophylaxis with lowmolecular-weight heparin.

LOD was assessed by CT scan without the use of dedicated software. A loss of domain between $20-50 \%$ was considered significant (Figures 1 and 2).

The intra-peritoneal catheter insertion was by a Seldinger technique in the left upper quadrant (LUQ). As the needle was advanced, fluid was infused to minimise the risk of puncturing the bowel. A "give" was felt when the fascia was pierced and then the guidewire was fed through the needle. The tract was then dilated to insert a double-lumen central venous catheter and the guidewire withdrawn. The daily volume insufflated was tailored to the patient's response 

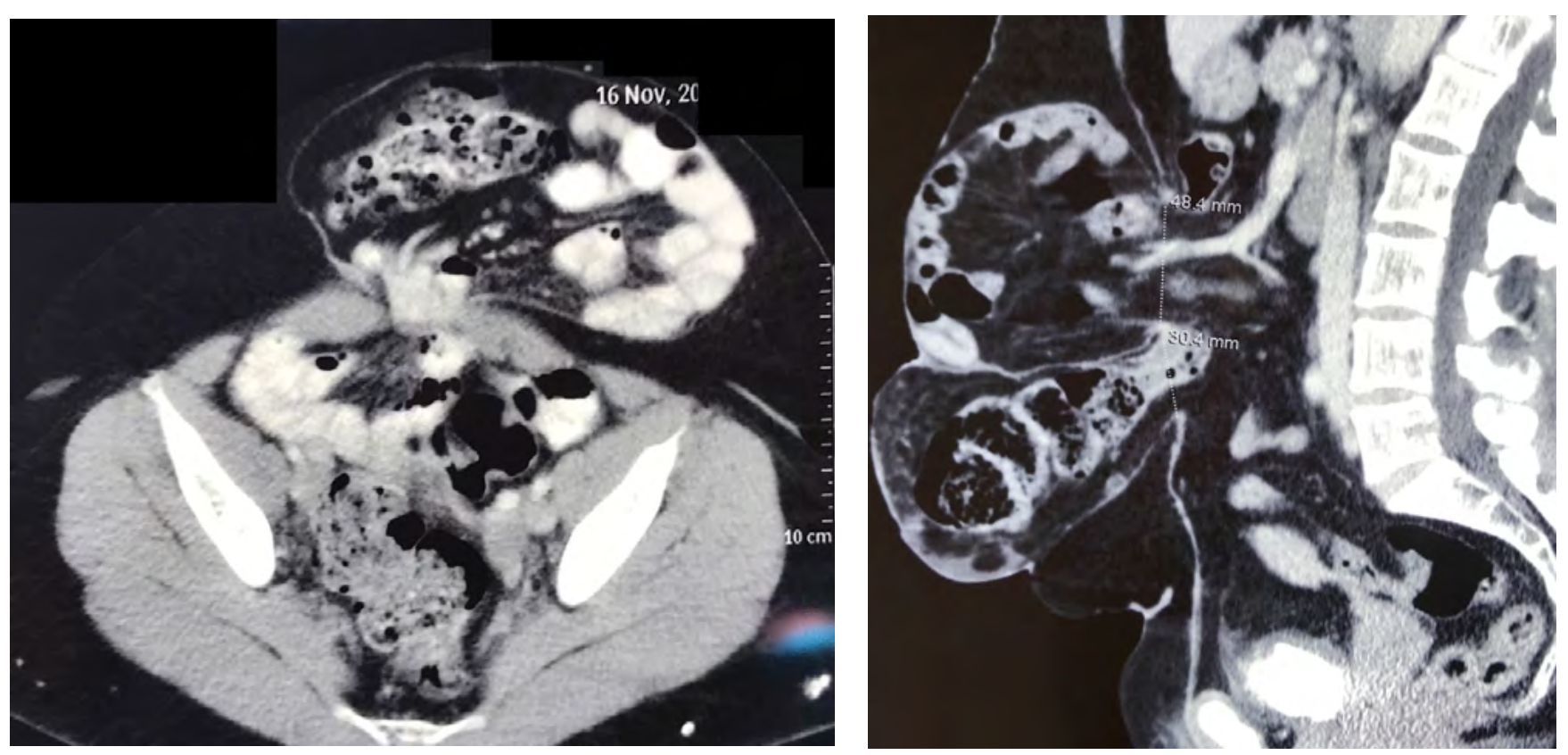

Figures 1 and 2: Pre-PPP axial and sagittal CT scans showing a calculated 50\% loss of domain

expressed as abdominal discomfort and shoulder blade pain. The duration of insufflation was between 10-14 days. Postinsufflation $\mathrm{CT}$ scan was obtained to evaluate the restoration of domain (Figure 3).

\section{Principles of insufflation technique}

The prerequisite to surgery is to insufflate the abdominal cavity with ambient air to address the content/container mismatch evidenced by constant pre-deflation and postclosure ratio. Preoperatively, the resultant pneumatic anti-oedema effect of air due to PPP combined with the compliant abdominal wall is expected to keep the peak airway pressure (PAP) within normal limit. Likewise, intraoperatively, the deflated abdomen combined with the reduced intra-abdominal volume post-closure of defect is also expected to keep the ratio unchanged since both the numerator and denominator are decreased. The identical pre-deflation and post-closure ratio together with normal

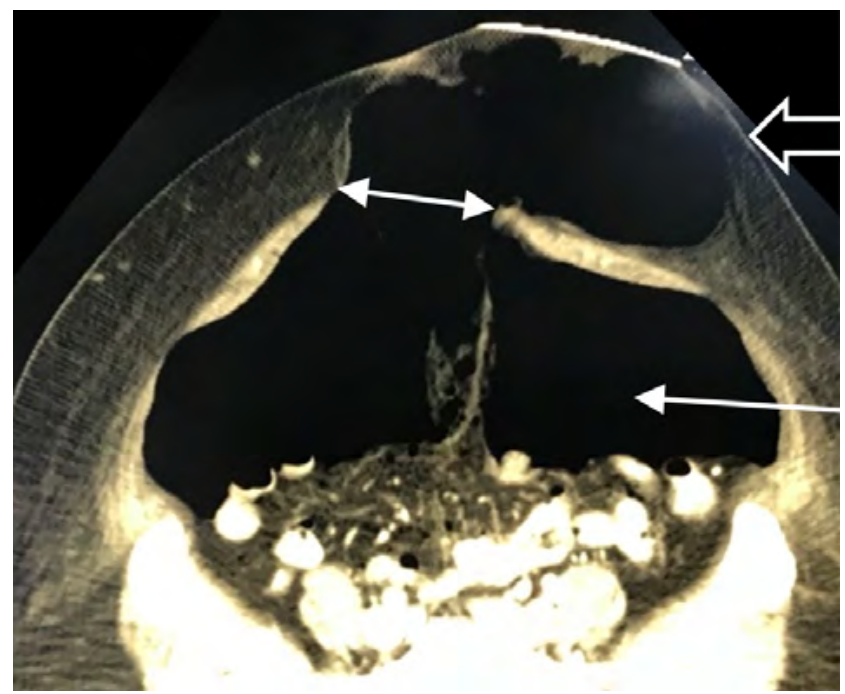

Figure 3: After PPP - note $>20 \%$ of LOD (large arrow), defect (double thin arrow), significantly reduced volume of intra-abdominal viscera (thin arrow)
PAP are the predictors of tension-free repair of hernia and form the basis of the IT.

Pre-deflation R1: Content/container ratio

Post-closure R2: Content/container ratio

PAP was recorded from the monitor on a relaxed patient ventilated on volume control mode.

A PAP under $35 \mathrm{~cm} \mathrm{H}_{2} \mathrm{O}$ should not be exceeded post closure of the defect.

Data collected include demographics, body mass index (BMI), comorbidities, indication for previous laparotomy, defect size, degree of LOD volume, type of air insufflated, complications related to the catheter insertion and the result of the pre- and post-insufflation computerised tomography scan (CT scan). The type of repair, ease of approximation of the linea alba, intraoperative pre-deflation and post-closure PAP, need for relook laparotomy for compartment syndrome, ICU admission, and local and systemic complications were also noted.

\section{Statistical analysis}

This is a descriptive observational study. Continuous variables are presented as mean, whereas categorical variables are expressed as frequencies (numbers, proportion or percentage). Data were obtained from theatre registry and files from the department of records and were captured in an Excel spreadsheet. Statistical analysis was performed with the statistical programme STATA 15.

\section{Results}

There were 16 females and three males. The mean age was 46.6 years (range 30-68). The causes of previous laparotomy were gynaecological, surgical and unknown in ten, three and two cases respectively. Primary ventral hernia was diagnosed in four cases. There were 12 patients with no previous repair and three patients had up to two repairs. The mean BMI was $33.32 \mathrm{~kg} / \mathrm{m}^{2}$ and $17 / 19$ patients were obese. The mean volume of air insufflated was 7.3 litres 


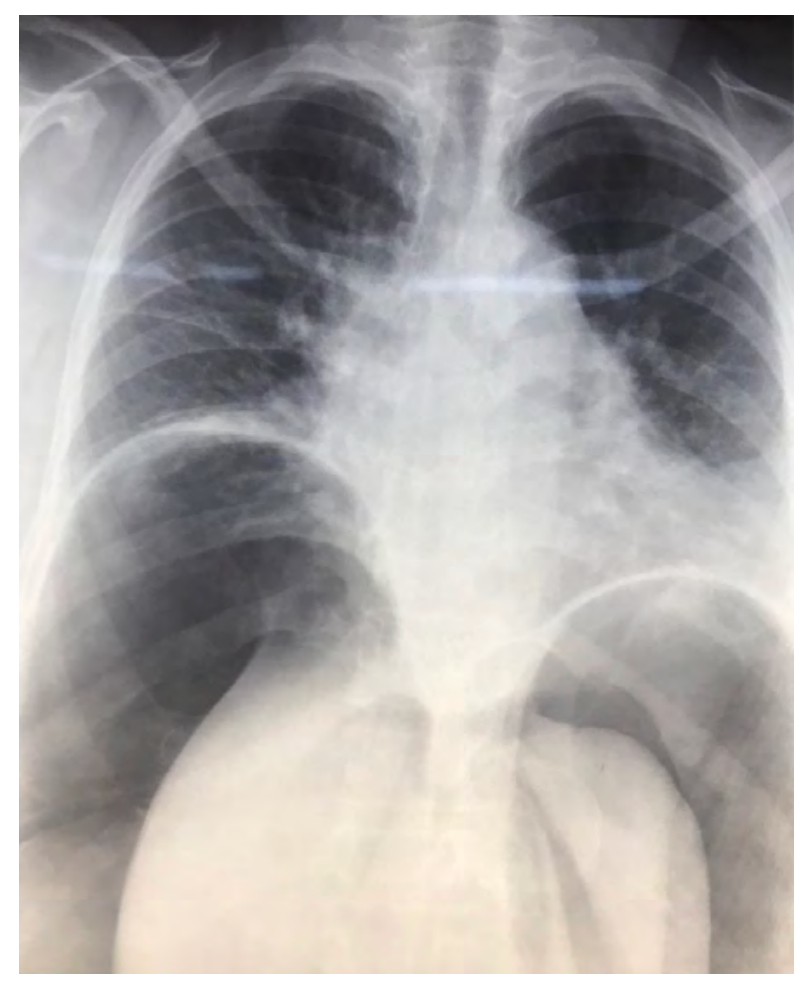

Figure 4: Post-PPP chest radiograph illustrates a significant pneumoperitoneum, splinting of the diaphragm and atelectasis

(range 5.5-12.4). The mean defect size was $15.8 \mathrm{~cm}$ (7-25). Atelectasis from the PPP is shown in Figure 4. The mean pre-deflation and post-closure PAP were 21 and $20 \mathrm{~cm} \mathrm{H}_{2} \mathrm{O}$ respectively, confirming that $\mathrm{R} 1$ and $\mathrm{R} 2$ are nearly the same. There were three instances of surgical site complications, two surgical site sepsis and one abdominal wall hematoma. The intraperitoneal catheter was inserted uneventfully in all cases. Only one patient spent a day in ICU due to an unexpected intraoperative bronchospasm. In one other case, there was an intraoperative iatrogenic injury of the herniated bowel and floppy bladder that were successfully repaired.

Simple tension-free midline approximation with combined continuous loop polydioxanone suture (PDS) and interrupted 1/0 PDS was achieved in 17 patients assessed to have a good quality myofascial complex. Two patients required myofascial release and underlay Permacol to achieve approximation of linea alba and to reinforce the weak aponeurosis respectively. Redundant skin was excised in all cases. One patient had panniculectomy. Portovac or pencil drains were inserted in 10 cases. There were no 30-day recurrences. There was no clinical evidence of compartment syndrome, DVT or pulmonary embolism.

\section{Discussion}

There is scant literature on PPP to facilitate the primary closure of ventral hernias with LOD and none from subSaharan Africa. Using the Goni Moreno protocol, Mancini et al. achieved fascial closure rate of $95.7 \%$ in a retrospective analysis of 162 cases from 1974 to $2019 .{ }^{9}$ A systematic review of the use of PPP by Martinez-Hoed et al. found 53 articles that included 1216 patients. ${ }^{10}$ Their review noted that the most common indication of PPP was large IH with LOD. In $99.6 \%$, the lost domain was reintroduced to the abdominal cavity and primary fascial closure was achieved in $86 \%$. The incidence of complications was $12 \%$, mainly minor, with five reported mortalities. The results reported in this article attest to the feasibility and low complication rate of this technique, and that it allows primary fascial closure in the vast majority of patients. Furthermore, it reduces the use of alloplastic materials that expose the patient to a small but lifelong risk of infection. ${ }^{11}$

\section{Conclusion}

IT enabled tension-free approximation of the linea alba in all cases of this subset of IH with significant LOD. Tissue repair was performed in most cases.

\section{Authors' contributions}

1. Conception and design - Dr I Bombil

2. Acquisition of data - Dr I Bombil

3. Analysis and interpretation of data - Dr I Bombil; Dr L Lushiku

4. Drafting of manuscript - Dr I Bombil; Dr L Lushiku

5. Critical revision - Dr I Bombil; Dr L Lushiku

6. Approval of the version to be published - Dr I Bombil; Dr L Lushiku

\section{Conflict of interest}

The authors declare no conflict of interest.

\section{Funding source}

No funding was required.

\section{Ethical approval}

Ethical approval was obtained from the Human Research Ethics Committee of the University of the Witwatersrand and the research review board of CHBAH (clearance certificate No. M200665).

\section{ORCID}

I Bombil (iD https://orcid.org/0000-0002-4819-0785 L Lushiku (iD https://orcid.org/0000-0002-8492-861X

\section{REFERENCES}

1. Mcadory RS, Cobb WS, Carbonell AM. Progressive preoperative pneumoperitoneum for hernias with loss of domain. Am Surg. 2009;75(6):504-8. https://doi. org/10.1177/000313480907500609.

2. Parker SG, Halligan S, Blackburn S, et al. What exactly is meant by "loss of domain" for ventral hernia? Systemic review of definitions. World J Surg. 2019;43(2):396-404. https://doi.org/10.1007/s00268-018-4783-7.

3. Sabbagh C, Dumont F, Fuks D, et al. Progressive preoperative pneumoperitoneum preparation (the Goni Moreno protocol) prior to large incisional hernia surgery: volumetric, respiratory and clinical impacts. A prospective study. Hernia. 2012;16:3340. https://doi.org/10.1007/s10029-011-0849-2.

4. Parida L, Pal K, Buainain HA, Elshafei H. Staged closure of giant omphalocele using synthetic mesh. APSP J Case Rep. 2014;5(3):27.

5. Alyami M, Passot G, Voiglio E, et al. Feasibility of catheter placement under ultrasound guidance for progressive preoperative pneumoperitoneum for large incisional hernia with loss of domain. World J Surg. 2015;39(12):2878-84. https://doi.org/10.1007/s00268-015-3206-2. 
6. Raynor RW, Del Guercio LR. The place of pneumoperitoneum in the repair of massive hernia. World J Surg. 1989;13(5):5815. https://doi.org/10.1007/BF01658874.

7. Mayagoitia JC, Suarez D, Arenas JC, Diaz de Leon V. Preoperative progressive pneumoperitoneum in patients with abdominal wall hernias. Hernia. 2006;10:213-7. https://doi.org/10.1007/s10029-005-0040-8.

8. Grabowski J, Talamini MA. Physiological effects of pneumoperitoneum. J Gastrointest Surg. 2008;13(5):1009-16. https://doi.org/10.1007/s11605-008-0662-0.

9. Mancini A, Mougin N, Venchiarutti V, et al. Goni Moreno progressive preoperative pneumoperitoneum for giant hernias: a monocentric retrospective study of 162 patients. Hernia. 2020;24:545-50. https://doi.org/10.1007/s10029-01902113-5.

10. Martinez-Hoed J,Bonafe-Diana S, Bueno-Liedo J.Asystematic review of the use of progressive preoperative pneumoperitoneum since its inception. Hernia. 2021;25(6):1443-58. https://doi.org/10.1007/s10029-020-02247-x.

11. Seker D, Kulacoglu H. Long-term complications of mesh repairs for abdominal-wall hernias. J Long Term Eff Med Implants. 2011;21(3):205-18. https://doi.org/10.1615/ JLongTermEffMedImplants.v21.i3.40. 